\title{
Stefan Hauser, Roman Opiłowski \& Eva L. Wyss (Hg.). 2019. Alternative Öffentlichkeiten. Soziale Medien zwischen Partizipation, Sharing und Vergemeinschaftung (Edition Medienwissenschaft. Band 35). Bielefeld: transcript Verlag. $318 \mathrm{~S}$.
}

Besprochen von Prof. i.R. Dr. Jürgen Schiewe: Universität Greifswald, Institut für Deutsche Philologie, Rubenowstr. 3, D-17487 Greifswald, E-Mail: jschiewe@uni-greifswald.de

https://doi.org/10.1515/zrs-2021-2079

Wenn es einem nicht schon beim Lesen des Titels dieses Buches aufgefallen ist, dann sieht man es spätestens beim Eintippen in ein Textverarbeitungsprogramm: Die Rechtschreibkorrektur macht darauf aufmerksam, dass es den Plural Öffentlichkeiten im Deutschen offenbar gar nicht gibt. Das Wort wird - z. B. von Word mit einer roten Wellenlinie markiert und als Korrekturvorschlag erscheint der Singular „Öffentlichkeit“. Ein Blick in einschlägige Wörterbücher bekräftigt (dieses Mal) den Hinweis der Textverarbeitung: Der Plural wird zumeist nicht explizit genannt, Belege mit dem Plural werden nicht angeführt und wenn doch vom Plural die Rede ist, dann heißt es, er sei „ungebräuchlich“. Damit bestätigen auch neuere Wörterbücher ${ }^{1}$ das, was bereits Johann Christoph Adelung („Die Öffentlichkeit, plur. inus.“) 1798 und Joachim Heinrich Campe („Die Öffentlichkeit. o. Mz.“) 1809 in ihren Wörterbüchern ${ }^{2}$ verzeichnet hatten - zu einer Zeit, als das Wort Öffentlichkeit im Deutschen als Übertragung von Publicität überhaupt erst gebräuchlich wurde. Anfang des 19. Jahrhunderts entwickelte sich Öffentlichkeit von einem Wort zu einem Begriff ${ }^{3}$ im Sinne von „Raum, in dem sich die öffentliche Meinung bildet und mit dem Anspruch eines Gegengewichtes zur staatlichen Herrschaft auftritt“ (Schiewe 2004: 61). Seither wird der Begriff üblicherweise nur im Singular verwendet und damit ausgedrückt, dass es innerhalb eines Staates nur einen solchen Raum mit nur einer öffentlichen Meinung gibt.

Nun ist es aber nicht nur aus Sicht der Wortbildung durchaus möglich, den Plural Öffentlichkeiten zu bilden, es kann sogar nötig werden, ausdrücklich von „Öffentlichkeiten“ zu sprechen - dann nämlich, wenn in mehreren Räumen mehrere Meinungen gebildet werden, die, wie es in den einleitenden Anmerkungen der HerausgeberInnen $\mathrm{zu}$ dem hier $\mathrm{zu}$ besprechenden Sammelband heißt, mit

1 Das DWDS und das online Duden-Wörterbuch verzeichnen zwar den Plural, führen aber keine Beispielbelege unter Verwendung des Plurals an.

2 Adelung 1793-1801, Bd. 3 (1798): 586 und Campe 1807-1811, Bd. 3 (1809): 550.

3 Zur Wort- und Begriffsgeschichte vgl. Hölscher 1978; 1979. Vgl. auch die kurze Zusammenfassung in Schiewe 2004: 44-55. 
dem Anspruch „einer eigenen öffentlichkeitswirksamen Macht“ (S. 11) auftreten. ${ }^{4}$ Es verwundert nicht, dass es die sozialen Medien sind, die die Existenz mehrerer Öffentlichkeiten nahelegen, denn sie haben das Gefüge von privater und öffentlicher Kommunikation grundlegend verändert und damit für sichtbare „Verschiebungen in der Tektonik von Öffentlichkeit“ (Hahn u.a. 2015: 13; vgl. S. 7) gesorgt.

Ausgangspunkt dieses von den ausgewiesenen MedienlinguistInnen Stefan Hauser, Roman Opiłowski und Eva L. Wyss herausgegebenen Sammelbandes ist die unbestreitbare Feststellung, dass die Digitalisierung von Medien und Kommunikationsformen dazu beigetragen hat, „neue Handlungsspielräume“ zu eröffnen, durch die „neue Formen der Partizipation an Öffentlichkeit“ ermöglicht worden sind (S. 7). Auch ihren Annahmen, „dass Öffentlichkeit stets Bestandteil sozialer Praktiken (gewesen) ist und dass sich die Praxis des Öffentlichen in der modernen Gesellschaft jeweils auch in Abhängigkeit von den medialen Bedingungen zeigt, denen sie unterliegt“ (S. 7), ist sicherlich zuzustimmen. Irritierend allerdings wirkt zweierlei: Erstens die Aussage, es dränge sich die Frage auf, „was Öffentlichkeit eigentlich ist“ (ebd., Hervorhebung von mir), und zweitens der Umstand, dass sich die theoretische Erörterung des Begriffs Öffentlichkeit darin erschöpft, ihn als „komplexes Phänomen“ (S. 8) zu bezeichnen. Es gibt keine „eigentliche“ Öffentlichkeit, denn Öffentlichkeit wird - wie es zutreffend heißt - „in Abhängigkeit von den medialen Bedingungen“ (S. 7) hergestellt. Genau diese wesentliche Eigenschaft von Öffentlichkeit hätte deutlicher herausgearbeitet werden sollen und können - sofern die HerausgeberInnen bereit gewesen wären, sich in die Geschichte des Begriffs zu vertiefen und die aufgeworfene Frage nach der Existenz und Beschaffenheit ,alternativer Öffentlichkeiten“ aus historischer Perspektive $\mathrm{zu}$ begründen und $\mathrm{zu}$ beleuchten.

In der Folge wird der Begriff „alternative Öffentlichkeit“ lediglich angebunden an die Formen von Medien, die konstitutiv für die Herstellung von Öffentlichkeit sind. In Abgrenzung zum Begriff „Gegenöffentlichkeit“, der eine „gegen eine hegemoniale Öffentlichkeit gerichtete Teilöffentlichkeit“ (S. 9) meint, heben die HerausgeberInnen für „alternative Öffentlichkeit“ die Tatsache hervor, „dass digitale Öffentlichkeiten auch durch nicht-institutionelle Akteure geschaffen werden können und mit teilweise anderen Produktions- und Rezeptionslogiken verbunden sind, als dies bei journalistischen Massenmedien (unter den Rahmenbedingungen des vordigitalen Zeitalters) der Fall war“ (S. 10). Anknüpfend an einen Öffentlichkeitsbegriff, der in den etablierten journalistischen Massenmedien eine die drei Staatsgewalten kontrollierende vierte Gewalt sieht, werden die sozialen

4 Mit Fokus auf derartige Zusammenhänge ist der Begriff „Öffentlichkeiten“ seit ungefähr zwanzig Jahren insbesondere in der Öffentlichkeitssoziologie gebräuchlich. 
Medien als eine „fünfte Gewalt“ bezeichnet, „die aus den vernetzten Vielen besteht und zu einer eigenen öffentlichkeitswirksamen Macht geworden ist“" (S. 11). Das anschließende Loblied darauf, welche positive Arbeit die Akteure dieser fünften Gewalt leisten - sie betreiben ein Agenda-Setting „,von unten“, erschaffen gut informierte alternative Öffentlichkeiten, treten als Medienkritiker und Meinungskorrektiv auf, bilden Protestgemeinschaften, machen Entlarvungs- und Enthüllungsarbeit, bringen politische Akteure und Unternehmen in Bedrängnis (vgl. S. 11) - soll angesichts politisch auch ganz anders einzuordnender Akteure und deren Nutzung sozialer Medien wohlwollend als idealistisch bezeichnet werden. Es bleibt zu prüfen, ob diese Form von Idealismus in den nachfolgenden Beiträgen geteilt oder auch problematisiert wird.

Der Sammelband enthält elf Beiträge, die nach Auskunft der HerausgeberInnen „nicht einem einheitlichen Konzept von alternativer Öffentlichkeit verpflichtet sind“, wohl aber alle „von der Frage ausgehen, wie sich die jüngeren Entwicklungen der Nutzung und sprachlichen Gestaltung der ,neuen“ Kommunikationsmöglichkeiten auf die Tektonik von Öffentlichkeit(en) auswirken“ (S. 12). Den Anfang macht der Beitrag „Diskurspragmatische Perspektiven auf neue Öffentlichkeiten in Webforen“ von Steffen Pappert und Kersten Sven Roth. Mit ihrer reflektierten Übersicht zu „Online- und Offline-Öffentlichkeiten“ füllen die Autoren eine in den einleitenden Anmerkungen vorhandene Lücke. Sie stellen in der Kommunikationswissenschaft genutzte Modelle von Offline-Öffentlichkeit(en) (Diskursmodelle, systemtheoretisches Spiegelbildmodell) vor und charakterisieren, teilweise unter Rückgriff auf deren Kategorien und Funktionsbeschreibungen, anschließend Online-Öffentlichkeit(en) im Web 2.0. Neben eher die traditionellen journalistischen Medien erweiternden Merkmalen, wie sie Foren oder Weblogs aufweisen, zeigen „persönliche Öffentlichkeiten“, erzeugt z.B. auf Facebook oder Twitter, ganz neue Qualitäten. Im Gegensatz zur Face-to-Face-Kommunikation sind sie „persistent“, „duplizierbar“, „skalierbar“ und „durchsuchbar“ (vgl. S. 31). Pappert und Roth erliegen nun nicht dem Idealismus von „Netzeuphorikern“, sondern erkennen in vielen Fällen praktizierter persönlicher Öffentlichkeit problematische Strukturen und Inhalte, die Roth (2017) als „Stammtisch-Modus“ bezeichnet hat:

\footnotetext{
„Mit der durch das Netz begünstigten Ausweitung und Aufwertung des Stammtisch-Modus, also einer Melange aus verbalisiertem Halbwissen, Vorurteilen und Wut, gehen Gefahren für die demokratisch-pluralistische Öffentlichkeit einher, die im Moment nur bedingt kalkulierbar sind.“ (S. 32)
}

Das sich daran anschließende Modell (diskurs)pragmatischer Basisbedingungen wird in einer exemplarischen Skizze erprobt an auf einen Spiegel-Online-Artikel folgenden Forenbeiträgen. Die auf verschiedenen sprachlichen Ebenen vorgenommene Analyse wird überzeugend vorgeführt, so dass sich das Modell als 
tragfähig erweist und seine Anwendung auf andere und umfangreichere Forenkommunikationen erfolgversprechend scheint.

In seinem Beitrag „Medien, Wahrnehmung, Öffentlichkeit. WahrnehmungsGemeinschaften und deren Interaktion als Gegenstand der Medienlinguistik“ geht GERD AnTos davon aus, dass Medienangebote „nicht nur Kommunikationsprozesse auslösen, sondern auch Gemeinschaften konstituieren und stabilisieren können“ (S. 54), und zwar „Wahrnehmungs-Gemeinschaften“, die Gegenstand einer „Linguistik der Wahrnehmung“ sein sollten. An einer Sammlung von historischen und gegenwärtigen Medien und der Praxis ihrer Handhabung sucht er aufzuzeigen, wie diese Wahrnehmungs-Gemeinschaften Öffentlichkeit(en) konstituiert und modifiziert haben, bis hin zu ihrer „Atomisierung“ durch die Macht von Big Data. Die eingangs angekündigte Analyse von „Leichter Sprache“, „Erregungsgemeinschaften“ und „Big-Data-Metadaten“ (S. 55f.) als Formen von „alternative (Gegen)Öffentlichkeit“ ausbildenden Wahrnehmungs-Gemeinschaften fällt am Ende (S. 71-74) leider allzu knapp und bloß allgemein aus.

Stringent, ja geradezu spannend liest sich der mit viel Beispielmaterial illustrierte Beitrag „Medien als Schutz vor Interaktionen“ von Ruтн Ayaß. Medien werden zumeist unter dem Aspekt betrachtet, dass sie Abwesenheit überbrücken und Interaktion zwischen nicht ko-präsenten Partnern ermöglichen können. Die Autorin interessiert sich für einen anderen Aspekt von Medien, für ihre Funktion, „Abwesenheit zu erzeugen“ (S. 82), also im Sinne Goffmans als involvement shields zu wirken - eine Eigenschaft, die offenbar zunehmend in öffentlichen Kontexten anzutreffen ist. Theoretisch und systematisch, historisch und kulturell werden Formen, Situationen und Strategien beschrieben, wie die eigene Anwesenheit durch den Gebrauch von Medien minimiert, auf jeden Fall modifiziert wird. Dieser Beitrag bietet zwar kaum Einsichten in alternative Öffentlichkeiten, dafür aber umso mehr Erkenntnisse und Anregungen zu alternativen Funktionen von Medien, gerade auch des Mobiltelefons, in der Öffentlichkeit.

Am Fallbeispiel der Reaktionen im Social Web (Facebook, Twitter) auf den Absturz des Airbus Flug 9525 von Barcelona nach Düsseldorf am 24. März 2015 untersucht Konstanze Marx „Kollektive Trauer 2.0 zwischen Empathie und Medienkritik“. Die dort geübte „kollektive Online-Trauerarbeit“ wird als eine Form „alternativer Öffentlichkeit“ bezeichnet, in der „sich der Umgang mit einem Tabuthema wieder deutlich einer größeren Gemeinschaft“ (S. 111) öffnet. Während man sich fragen kann, warum Menschen überhaupt ihre - tatsächlich empfundene oder möglicherweise auch geheuchelte - Trauer im Netz glauben artikulieren zu müssen (um ihre Existenz nachzuweisen?), beschreibt die Autorin die „Trauergemeinde 2.0" als eine medienkritische Gemeinschaft, die die Kriterien der angemessenen Trauerkommunikation selbst aushandelt und ein den Trauerkreis erweiterndes „Trauerarchiv“ (S. 126) geschaffen hat. 
Was unter alternativen Öffentlichkeiten verstanden werden kann, welche gesellschaftspolitische Funktion sie haben und welche Veränderungen sie bewirken können, zeigen Birte C. Gnau und Eva L. Wyss in ihrem Beitrag „Der \#MeTooProtest. Diskurswandel durch alternative Öffentlichkeit“. Anders als bei Trauerinszenierungen werden soziale Medien bei diesem bedrückenden Thema genutzt, um Missbrauchserfahrungen individuell und zugleich kollektiv zu artikulieren und auf diese Weise „den Wunsch nach Unterstützung, Trost und Sich-Öffnen-Können“ (S. 136) kundzutun. Die Autorinnen zeigen überzeugend auf, dass die in den sozialen Medien geschaffene alternative Öffentlichkeit der angemessene Raum für dieses „Sich-Öffnen-Können“ war (und natürlich immer noch ist) und dass erst durch sie ein Diskurswandel in den traditionellen Leitmedien ausgelöst wurde. Es ist das Verdienst dieses Beitrags, eine derart relevante, weil emanzipatorische und aufklärerische und hoffentlich auch Strukturen verändernde Funktion von in sozialen Medien geschaffener alternativer Öffentlichkeit sichtbar zu machen.

Christine Domke interessiert sich in ihrem Beitrag „Meso-Kommunikation zwischen Stadt/Netz: Zur Struktur alternativer Kommunikationswege“ für die Frage, wie sich die zwischen Face-to-Face- und Massenkommunikation anzusiedelnde Meso-Kommunikation im öffentlichen Raum (Plakate, Aufkleber, Durchsagen, Leitsysteme, Hinweisschilder u.v.m.; vgl. die Übersicht S. 173f.) zu Formen der Meso-Kommunikation online verhält. Sie zeigt, dass „Adressabilität, eine spezifische Rezipientenauswahl und medial-materiale Organisiertheit“ (S. 181) sowohl Merkmale von lokalen Textwelten und Kommunikationen sind als auch von digitalen gruppenhaften Vernetzungen auf Facebook und Twitter. Wie gerade die sozialen Medien eine meso-kommunikative alternative Öffentlichkeit schaffen und damit zugleich eine Konvergenz lokaler und massenmedialer Wahrnehmung und Kommunikation bewirken können, wird am Beispiel einer Positionierung zur Flüchtlingsthematik überzeugend beschrieben.

Tномаs Metтen beschäftigt sich in seinem Beitrag „Webvideos als Medien öffentlicher Wissenschaft. Eine exemplarische Analyse audiovisueller Strategien der Wissenschaftskommunikation“ mit einem bislang wenig beachteten Gegenstand: der digitalen Präsentation wissenschaftlicher Erkenntnisse als Ausdruck gewandelter Legitimationsanforderungen an die Wissenschaft. Exemplarisch untersucht wird ein Webvideo aus dem Bereich der Biologie, in dem Darstellen und Vermitteln als grundlegende Funktionen populärer Wissenschaftskommunikation erkennbar werden. Der Autor weist in seiner präzisen Bild-Text-Analyse nach, dass sich in dem Webvideo eine adressatenorientierte Inszenierung von Wissenschaft und eine sachorientierte Erklärung überlagern (vgl. S. 212) und die auf diese Weise konstituierte Wissenschaftsöffentlichkeit ein durch das Medium geprägtes, nirgendwo sonst vorhandenes, ein „filmförmiges Wissen“ (S. 214) vermittelt bekommt. 
Françoise Hammer beschäftigt sich in ihrem Beitrag „Formen der Partizipation online. Dissens in der Leserrezension" mit erst durch das Internet möglich gewordenen Texten: den Laienrezensionen zu Büchern auf den Bestellseiten von Onlinehändlern. Anhand eines konkreten Beispiels werden Sprachhandlungsmuster aufgezeigt, mit denen Dissens als Abgrenzung zu einer allgemein vertretenen Meinung ausgedrückt, in diesem Fall das betreffende Buch negativ bewertet wird. Die durch Dialogisierung, Subjektivierung und Narrativierung gekennzeichneten Äußerungen - sollte man wirklich von „Rezensionen“ sprechen? bleiben in aller Regel autonom und monologisch, nur gelegentlich lösen sie eine Interaktion aus. Diese Form kommerziell inszenierter Kommunikation als „alternative Öffentlichkeit der Laienrezensenten“ (S. 233) zu bezeichnen, erscheint vor dem Hintergrund der Analyseergebnisse kaum angebracht.

Ein kontrastives Vorgehen wählt Roman Opıєowsкı in seinem Beitrag „Thematische Felder in deutschen und polnischen Online-Kommentaren zum Terroranschlag in Nizza im Juli 2016“. Eingangs führt der Autor in die „Problematik von Öffentlichkeit“ (S. 237ff.) ein und benennt für eine alternative Öffentlichkeit „im Kontext internetbasierter Kommunikationsformen“ drei Hauptmerkmale: „Flüchtigkeit der Kommunikationsteilnahme“, „Atomisierung der Kommunikationsgebiete“ und „Zugänglichkeit des Kommunikationsraumes“ (S. 241). Im Anschluss führt er eine qualitative Diskursanalyse von Nutzerkommentaren zu dem genannten Terroranschlag auf den Facebook-Profilen von Der Spiegel und Polity$k a$ durch. Die zunächst einzelsprachlich gewonnenen Ergebnisse werden abschließend gegenübergestellt und Gemeinsamkeiten und Unterschiede beschrieben. Am Ende steht die Behauptung, dass die Nutzerkommentare eine zunehmend bedeutsam werdende alternative Online-Öffentlichkeit bilden, weil dort „relevante Räume der Repräsentanz und Meinungsbildung von Gesellschaften“ (S. 266) entstanden sind.

AndReas Osterroth reflektiert zu Beginn seines Beitrags „Sprache-Bild-Kommunikation in Imageboards. Das Internet-Meme als multimodaler Kommunikationsakt in alternativen Öffentlichkeiten“ über den Begriff Öffentlichkeit und - ein Alleinstellungsmerkmal in diesem Band - die Frage, warum er überhaupt im Plural verwendet werden kann. Imageboards, auf denen mittels Memes kommuniziert wird, schaffen „alternative Diskurse“, sind „basisdemokratisch organisiert“ und bilden nach Ansicht des Autors deshalb auch eine „alternative Öffentlichkeit“. Im weiteren Verlauf erhält man eine grundlegende Einführung in das Thema „Memes" und eine Erläuterung ihrer Funktionen und normativen Regeln. Der Beitrag endet mit der gewichtigen und aufschlussreichen Aussage, dass in Ländern, in denen die traditionelle Öffentlichkeit reglementiert wird, ,das Imageboard als alternative Öffentlichkeit ein Hort der Demokratie“ ist. Dort ist nur erfolgreich, „was die Nutzer der speziellen Öffentlichkeit im Schwarm so bewerten“ (S. 284). 
Der Band wird abgeschlossen mit dem Beitrag „Kundenbeschwerden in der digitalen Öffentlichkeit als Form des Widerstands - am Beispiel des Facebookauftritts von Vodafone“ von NAdine Rentel. Was vormals per Brief, Telefonat oder E-Mail vollzogen wurde, die Beschwerde bei einem Unternehmen, wird heute oftmals auf der Facebook-Seite des Unternehmens öffentlich kundgetan. Die Autorin untersucht in überzeugender Weise diese Beschwerdekommunikation hinsichtlich ihrer Funktionen, der in Beschwerden enthaltenen Teilhandlungen und der darin angewandten sprachlichen Strategien. Wie nicht anders zu erwarten, wird auch die in dem öffentlichen Raum eines sozialen Mediums produzierte Beschwerdekommunikation als „Form des Protests bzw. als Entwurf einer alternativen Gegenöffentlichkeit bewertet“ (S. 289; auf S. 308 die gleiche Formulierung mit der Einschränkung „[...] als Versuch des Entwurfs [...]“).

Der vorliegende Band bereichert durch eine Reihe kluger und überzeugend gearbeiteter Analysen unterschiedlicher Kommunikationsformen und thematischer Diskurse in sozialen Medien: Webforen, Webvideos, Meso-Kommunikation, Internet-Memes; Trauer, MeToo, Leserrezensionen, Online-Kommentare, Kundenbeschwerden. Hinzu kommen allgemeinere Überlegungen zu Wahrnehmungs-Gemeinschaften und zur Interaktionsverweigerung als Funktion von Medien. Die Analysen, die allesamt für sich den Anspruch auf Erkenntnisgewinn erheben können, werden von den AutorInnen in aller Regel zu Beginn und am Ende ihrer Beiträge gerahmt durch eine Einordnung in ein Konzept von alternativen Öffentlichkeiten, das aber sowohl für den gesamten Band als auch für die einzelnen Beiträge zu wenig reflektiert ist und deshalb auch kaum greifbar wird.

Genügt es für die Schaffung einer alternativen Öffentlichkeit bereits, dass eine Gruppe von Usern sich in einem sozialen Medium zu irgendeinem Thema äußert? Berechtigt der Umstand, dass diese Äußerungen in einem öffentlich zugänglichen sozialen Medium getätigt werden und sich inhaltlich im Gegensatz zu Äußerungen in traditionellen analogen Medien befinden, zu der Aussage, es handele sich um alternative Öffentlichkeiten? Ist es der Umstand, dass sich User, die keinen Zugang zu traditionellen Medien und damit zur Mitbestimmung über eine traditionelle Öffentlichkeit haben, in sozialen Medien äußern, durch den alternative Öffentlichkeiten konstituiert werden? Und: Bildet jedes Thema - Trauer über einen Flugzeugabsturz, Unzufriedenheit mit einem Roman oder einem Unternehmen - bereits eine alternative Öffentlichkeit? Spielt ein aufklärerischer, emanzipatorischer, ein politischer Impetus eine Rolle für alternative Öffentlichkeiten?

Diese und gewiss noch mehr Fragen müssten gestellt und theoretisch-systematisch erörtert werden, um den Begriff „alternative Öffentlichkeiten“ aus dem Bereich eines „wissenschaftlichen Schlagwortes“ in den eines wissenschaftlichen Begriffs mit Erkenntnispotential zu überführen. Die besten Ansätze dazu liefert der Beitrag von Birte C. Gnau und Eva L. Wyss über den „\#MeToo-Protest“. Hier 
wird nachvollziehbar, warum und wie eine alternative Öffentlichkeit sich bilden musste, um ein sowohl in der Privatheit als auch in der traditionellen Öffentlichkeit verschwiegenes, tabuisiertes Thema für einen immer größeren Kreis von unmittelbar und mittelbar Betroffenen wahrnehmbar zu machen. Es ist nicht angemessen, dieses gesellschaftspolitisch eminent wichtige Thema auf die gleiche Stufe zu stellen mit subjektiven, sagen wir: rasch formulierten Meinungsäußerungen über die Qualität eines Buches und beides als „alternative Öffentlichkeiten“ zu bezeichnen. Von den vielen anderen „Diskursen“ in sozialen Netzwerken mit rechtsradikalen, rassistischen, antidemokratischen, hetzerischen, hasserfüllten Inhalten wäre natürlich auch noch zu sprechen, wenn man den Begriff „alternative Öffentlichkeiten“ oder gar „alternative Gegenöffentlichkeiten“ nahezu ohne qualitatives Merkmal benutzt.

Trotz dieser kritischen Anmerkungen soll abschließend betont werden, dass die in dem besprochenen Sammelband enthaltenen Beiträge Anregungen geben, die Öffentlichkeitsgeschichte aus dem Blickwinkel des alternativen Potentials von digitaler Kommunikation, Interaktion und Medien(wandel) weiter und auch neu zu denken und zu beschreiben. Die lange Vorgeschichte analoger Öffentlichkeit und die ebenfalls lange Geschichte des Öffentlichkeitsbegriffs sollten dabei aber nicht einfach ignoriert werden.

\section{Literatur}

Adelung, Johann Christoph. 1793-1801. Grammatisch-kritisches Wörterbuch der Hochdeutschen Mundart, mit beständiger Vergleichung der übrigen Mundarten, besonders aber der Oberdeutschen. Zweyte vermehrte und verbesserte Ausgabe. 4 Bände. Leipzig: Johann Gottlob Immanuel Breitkopf und Compagnie.

Campe, Joachim Heinrich. 1807-1811. Wörterbuch der deutschen Sprache. 5 Bände. Braunschweig: Schulbuchhandlung.

Hahn, Oliver, Ralf Hohlfeld \& Thomas Knieper. 2015. Einführung: Digitale Öffentlichkeit - Digitale Öffentlichkeiten. In: Dies. (Hg.): Digitale Öffentlichkeit(en). München: UVK Verlagsgesellschaft, 11-18.

Hölscher, Lucian. 1978. Öffentlichkeit. In: Otto Brunner, Werner Conze \& Reinhart Koselleck (Hg.). Geschichtliche Grundbegriffe. Historisches Lexikon zur politisch-sozialen Sprache in Deutschland. Bd. 4. Stuttgart: Klett-Cotta, 413-467.

Hölscher, Lucian. 1979. Öffentlichkeit und Geheimnis. Eine begriffsgeschichtliche Untersuchung zur Entstehung der Öffentlichkeit in der frühen Neuzeit. Stuttgart: Klett-Cotta.

Roth, Kersten Sven. 2017. „Stammtisch 2.0“? Politiker-Kritik als interaktionale Ressource in Webforen. In: Heidrun Kämper \& Martin Wengeler (Hg.). Protest - Parteienschelte - Politikverdrossenheit. Politikkritik in der Demokratie. Bremen: Hempen, 77-97.

Schiewe, Jürgen. 2004. Öffentlichkeit. Entstehung und Wandel in Deutschland. Paderborn: Schöningh. 